SCIENTIFIC REPORT

\title{
Steroid prophylaxis in eyes with uveitis undergoing phacoemulsification
}

\author{
W R Meacock, D J Spalton, L Bender, R Antcliff, C Heatley, M R Stanford, E M Graham
}

Br J Ophthalmol 2004;88:1122-1124. doi: 10.1136/bjo.2003.032482

\begin{abstract}
Aim: To compare the efficacy of two preoperative steroid regimens for cataract surgery in patients with uveitis.

Methods: 40 uveitis patients with cataract underwent phacoemulsification and intraocular lens (IOL) implantation. Preoperatively they were randomised into two groups: group 1 (20 patients) received a single dose of intravenous methylprednisolone $(15 \mathrm{mg} / \mathrm{kg}$ ) half an hour before surgery, and group 2 (20 patients) received a 2 week course of oral prednisolone $(0.5 \mathrm{mg} / \mathrm{kg})$ which was tapered postoperatively. Preoperatively patients had aqueous flare and cells measured with the Kowa laser flare meter. On days 1, 7, 28, and 90 aqueous flare and cells were measured, and on days 7 and 90 fluorescein angiography was performed to determine the incidence of cystoid macular oedema (CMO). Results: At all postoperative visits the mean increase in flare was greater for group 1 (intravenous steroid). Patients with posterior synechiae had greater blood-aqueous barrier damage $(B A B)$ postoperatively. There were no statistically significant differences in logMAR visual acuity and incidences of CMO between the two groups at 7 and 90 days. Conclusion: A 2 week course of oral prednisolone, tapered postoperatively, produced a better recovery of the BAB than a single dose of intravenous methylprednisolone and is thus the recommended preoperative regimen.
\end{abstract}

C ataracts occur in up to $50 \%$ of patients with uveitis and ocular inflammation should be minimised for at least 2 months before proceeding with surgery. ${ }^{1-3}$ Additionally, these patients should receive increased preoperative immunosuppressive steroid prophylaxis as this has been shown to increase the proportion of patients with a visual acuity (VA) of 20/40 or better at 3 months. ${ }^{3}$ The aim of this prospective randomised study was to compare the effects of a single dose of intravenous methylprednisolone given half an hour before surgery with a 2 week preoperative course of oral prednisolone on VA, blood-aqueous barrier recovery $(\mathrm{BAB})$, and the incidence of angiographic CMO in patients with uveitis undergoing phacoemulsification.

\section{PATIENTS AND METHODS}

Following ethics committee approval patients with uveitis requiring cataract surgery were randomised to two groups of preoperative augmented steroid treatment. Group 1 received intravenous methylprednisolone $(15 \mathrm{mg} / \mathrm{kg}$ given over half an hour before surgery) and group 2 had a 2 week course of oral prednisolone $(0.5 \mathrm{mg} / \mathrm{kg} /$ day $)$ before surgery. Attempts were made to minimise ocular inflammation for at least 2 months preoperatively using either topical or oral steroid.

On the day of surgery logMAR VA was measured and aqueous flare and cells were determined with the Kowa FC1000 laser cell flare meter (LCFM). Patients underwent clear corneal incision phacoemulsification and acrylic intraocular lens implantation by a single surgeon. Posterior synechiae were present in $40 \%$ of group 1 and in $58 \%$ of group 2 . The iris was stretched with Hirschmann hooks and iris sphincterotomies were performed in five and six patients in groups 1 and 2, respectively.

Postoperatively all patients used neomycin, polymixin, and dexamethasone $0.1 \%$ drops (Maxitrol) six times daily for 14 days and then continued with dexamethasone $0.1 \%$ (Maxidex) six times daily for 30 days. Patients were also treated with ketorolac (Acular) three times daily for 30 days. Group 1 patients receiving systemic immunosuppressive therapy before surgery continued their treatment according to the severity of ocular inflammation. Group 2 patients had their prednisolone dosage reduced by $5 \mathrm{mg}$ /day every week until their preoperative dosage was reached.

On days $1,7,28$, and 90 patients had their VA, aqueous flare, and cell counts measured. All patients had fluorescein angiography at the 7 and 90 day visit to determine the incidence of pseudophakic CMO.

\section{Statistical methods}

A sample of 40 (20 patients per group) was chosen to provide $80 \%$ power to detect a $20 \%$ difference in the change in postoperative aqueous flare with a two sided error at $\alpha=0.05$. The difference in preoperative flare values between the two groups was tested using Student's $t$ test. Univariate analyses were done on factors that were considered important in the delay in $\mathrm{BAB}$ recovery. In addition to the study group (intravenous methylprednisolone or oral prednisolone) and whether patients received systemic immunosuppressive therapy at the time of enrolment, other baseline factors were evaluated. Demographic variables were age, sex, and race. Ocular variables were type of uveitis and the presence of posterior synechiae. Mann-Whitney $U$ and Fisher's exact tests were used to test for differences in anterior chamber cells and postoperative CMO.

\section{RESULTS}

Twenty patients were enrolled in group 1 (intravenous methylprednisolone) and 20 to group 2 (oral prednisolone). Table 1 shows the characteristics of both groups of patients. Three patients in group 2 were excluded because of posterior capsule rupture during cataract surgery. The number of patients taking systemic immunosuppression at the time of enrolment was $10(50 \%)$ for group 1 and six (35\%) for group $2, \mathrm{p}=0.4$. Three patients in group 2 had weight gain and there were no complications in group 1.

Mean preoperative flare was 27.30 photons/ms (95\% CI, 12.1 to 39.6) for group 1 and 33.80 (95\% CI, 17.1 to 47.9 ) photons/ms for group $2(\mathrm{p}=0.2)$. At all postoperative visits

Abbreviations: $\mathrm{BAB}$, blood-aqueous barrier; $\mathrm{CMO}$, cystoid macular oedema; IOL, intraocular lens; LCFM, laser cell flare meter; VA, visual acuity 


\begin{tabular}{|lll|}
\hline Table 1 Patient characteristics & & \\
\hline & Group 1 & Group 2 \\
\hline Mean age & 47 & 52 \\
Sex ratio (M: F) & $8: 12$ & $12: 5$ \\
Ethnicity (W: AF: Pk: B)* (patient numbers) & $16: 3: 1: 0$ & $12: 3: 1: 1$ \\
Uveitis typet (patient numbers) & & \\
Idiopathic (A) & 6 & 6 \\
Idiopathic (B) & 4 & 3 \\
Ankylosing spondylitis (A) & 2 & 0 \\
Juvenile chronic arthritis (A) & 0 & 1 \\
Behçet's (P) & 2 & 0 \\
Behçet's (B) & 1 & 3 \\
Sarcoid (B) & 2 & 1 \\
Retinal vasculitis (P) & 1 & 0 \\
Pars planitis & 1 & 0 \\
Reiter's (A) & 1 & 1 \\
Sympathetic (B) & 0 & 1 \\
Toxoplasmosis (B) & 0 & \\
Multiple sclerosis (B) & 0 & \\
\hline *W, white; AF, African; Pk, Pakistani; Bg, Bangladeshi. & & \\
†A, anterior; P, posterior; B, both. & & \\
\hline
\end{tabular}

the mean increase in flare from preoperative value was greater for group 1 (fig 1). At 90 days, mean flare change was 8.88 photons $/ \mathrm{ms}$ (95\% CI, 2.9 to 16.1 ) for group $\mathrm{l}$ and 1.33 photons $/ \mathrm{ms}$ (95\% CI, 0.01 to 4.7$)$ for group $2(\mathrm{p}=0.05)$. At 90 days, the proportion of patients whose aqueous flare returned to preoperative levels was 13 (65\%) and 11 (73\%) for groups 1 and 2, respectively. Mean flare change for patients already receiving systemic immunosuppression at the time of study enrolment was 5.0 (95\% CI, 1.1 to 17.9) and 3.6 photons/ms (95\% CI, 0.1 to 8.7 ) at 28 and 90 days, respectively, and for those patients on topical steroid only was 4.9 (95\% CI, 2.2 to 16.3 ) and 6.0 photons/ms (95\% CI, 1.2 to 10.3$)(\mathrm{p}=0.75)$.

At 28 days, the median anterior chamber cell count change was 0 for patients of group 1 and 0.15 for group $2(p=0.2)$. At 90 days it was -0.2 and 0.2 for groups 1 and 2, respectively $(\mathrm{p}=0.6)$.

At 90 days VA improved in $16(80 \%)$ patients in group 1 and in $15(75 \%)$ patients in group $2(\mathrm{p}=0.8$, fig 2$)$. In group 1,13 patients $(65 \%)$ had a VA of 0.2 (Snellen 6/12) or better; $11(65 \%)$ of group 2 attained a VA of 0.2 or better. There were five patients in both groups who had VA worse than 0.2 attributable to posterior segment pathology. In group 1, three patients had a macular scar and two had pseudophakic CMO; in group 2 two patients had macular scar, two had pseudophakic CMO, and one had an epiretinal membrane.

Preoperatively, none of the patients had clinical CMO, but this may have been masked by cataract. At 1 week postoperative angiographic CMO was present in seven patients $(35 \%)$ in group 1 (five with panuveitis and two with anterior uveitis), and three (18\%) in group 2 (panuveitis in all three)

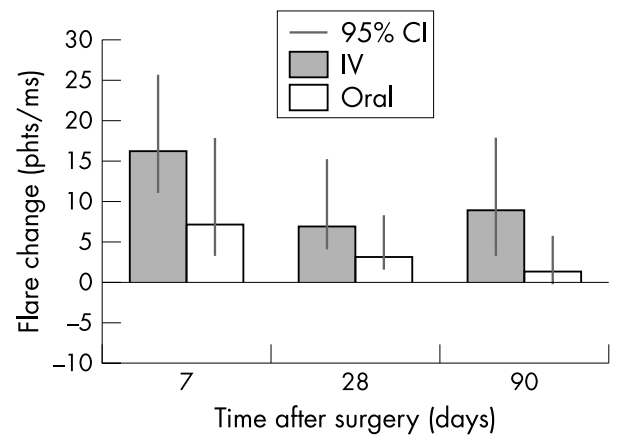

Figure 1 Mean postoperative flare.

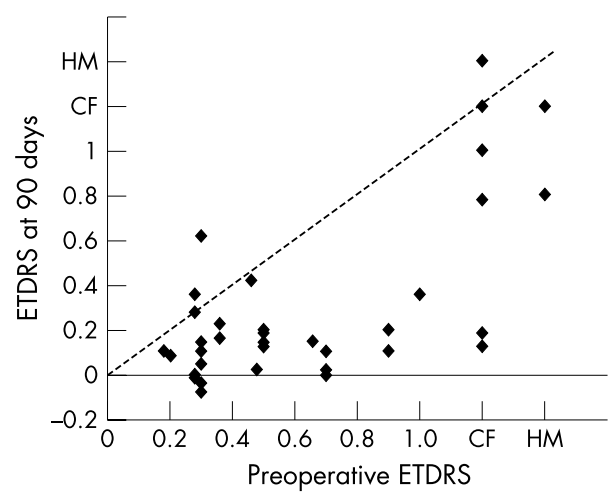

Figure 2 Preoperative and postoperative visual acuity.

$(\mathrm{p}=0.2)$; at 90 days angiographic CMO was present in six patients $(35 \%)$ of group 1 and five $(29 \%)$ in group $2(p=0.4)$. The treatment for clinical CMO (two patients in group 2) was orbital floor steroid injections (triamcinolone acetonide $40 \mathrm{mg}$,). The postoperative flare change was not significantly greater in these patients (table 2 ).

Exacerbation of uveitis occurred in two patients of both groups during follow up, which was treated with topical steroid.

\section{DISCUSSION}

This study compared $\mathrm{BAB}$ recovery and the incidence of angiographic CMO following cataract surgery in two groups of uveitis patients treated with different augmented preoperative immunosuppressive regimens.

The measurement of aqueous flare by LCFM has been shown to be an accurate and reproducible measurement of BAB damage. ${ }^{45}$ Preoperative flare varied among the patients of both groups although the mean difference between the groups was not significant. Accordingly, the changes from preoperative levels were used to measure the effect of surgery on the BAB postoperatively. At 28 and 90 days the decrease in flare from preoperative values was less for the intravenous group, reaching statistical significance at 90 days, indicating that there was less $\mathrm{BAB}$ damage in those patients who received oral steroid. This could have been due to the continued use of prednisolone during the period of steroid tapering, over 2-3 months following phacoemulsification. A possible explanation for greater flare change in group 1 could be a greater proportion of patients who had more acute 
Table 2 Univariate analysis on flare change data for all patients

\begin{tabular}{|c|c|c|c|c|}
\hline & \multicolumn{2}{|l|}{28 days } & \multicolumn{2}{|l|}{90 days } \\
\hline & \multicolumn{2}{|l|}{ Mean flare change } & \multicolumn{2}{|l|}{ Mean flare change } \\
\hline & photons/ms (95\% Cl) & p Value & photons/ms (95\% Cl) & p Value \\
\hline $\begin{array}{l}\text { Preoperative steroid } \\
\text { IV methyl prednisolone } \\
\text { Oral prednisolone } \\
\text { Uveitis type }\end{array}$ & $\begin{array}{l}6.93(3.8 \text { to } 13.4) \\
3.12(1.7 \text { to } 6.1)\end{array}$ & 0.16 & $\begin{array}{l}8.88(2.9 \text { to } 16.1) \\
1.33(0.01 \text { to } 4.7)\end{array}$ & 0.05 \\
\hline $\begin{array}{l}\text { Anterior uveitis } \\
\text { Posterior/panuveitis } \\
\text { Posterior synechiae }\end{array}$ & $\begin{array}{l}10.54(3.8 \text { to } 19.6) \\
2.94(0.6 \text { to } 4.9)\end{array}$ & 0.17 & $\begin{array}{l}4.54(0.7 \text { to } 8.1) \\
6.51(1.8 \text { to } 14.8)\end{array}$ & 0.35 \\
\hline $\begin{array}{l}\text { Present } \\
\text { Absent }\end{array}$ & $\begin{array}{l}12.94(5.7 \text { to } 17.4) \\
0.51(0.1 \text { to } 7.2)\end{array}$ & 0.007 & $\begin{array}{l}7.16(2.1 \text { to } 10.8) \\
4.41(0.6 \text { to } 9.5)\end{array}$ & 0.38 \\
\hline
\end{tabular}

attacks of uveitis before enrolment, but this was not evident from the records. There was no significant difference in anterior chamber cell count between the two groups at any time in the study. However, the LCFM measurement of anterior chamber cells is thought to be a less satisfactory measure of $\mathrm{BAB}$ damage as it registers all particles within the anterior chamber larger than $10 \mu \mathrm{m}$ and does not differentiate pigment granules (which may be released following pupil dilation) and leucocytes.

At 90 days 35\% patients in group 1 and 29\% in group 2 had angiographic CMO, which is higher than the $19 \%$ reported by Okhravi et al, who calculated the incidence of clinical CMO. ${ }^{3}$ The same study found an association between the severity of anterior uveitis and the incidence of clinical CMO, both of which were graded clinically. This relation was not reproduced in our study, which used an objective measurement of $\mathrm{BAB}$ breakdown.

At 90 days vision improved in $80 \%$ of patients in group 1 and $88 \%$ of patients in group 2 . Those patients who did not benefit from surgery either had pre-existing macula pathology or developed pseudophakic CMO.

Minimising BAB damage in uveitis patients is achievable through the use of modern surgical techniques and the use of biocompatible implants. Accepted practice has been that prophylactic augmented systemic immunosuppression should be given to patients with active uveitis before surgery. This study showed that a 2 week course of oral prednisolone tapered postoperatively was superior to a single intravenous bolus of steroid in minimising $\mathrm{BAB}$ damage. More work needs to be done, however, to define the ideal regimen, balancing the ocular benefits against the risk of systemic side effects.

\section{Authors' affiliations}

W R Meacock, D J Spalton, L Bender, R Antcliff, C Heatley,

M R Stanford, E M Graham, Tremona Road, Eye Unit, Southampton General Hospital, Southampton, Hants, UK

This work was presented at Association for Research in Vision and Ophthalmology 2000 and ESCRS Congress in Nice 2002.

Correspondence to: Mr W Meacock, Tremona Road, Eye Unit, Southampton General Hospital, Southampton, Hants, UK; wmeacock@ hotmail.com

\section{REFERENCES}

1 Hooper PL, Rao NA, Smith RE. Cataract extraction in uveitis patients. Surv Ophthalmol 1990;35:120-44.

2 Foster CS, Fong LP, Singh P. Cataract surgery and intraocular lens implantation inpatients with uveitis. Ophthalmology 1989;96:281-8.

3 Okhravi N, Lightman S, Towler HMA. Assessment of visual outcome after cataract surgery in patients with uveitis. Ophthalmology 1999;106:710-22.

4 Shah SM, Spalton DJ. Changes in anterior chamber flare and cells following cataract surgery. Br J Ophthalmol 1994;78:91-4.

5 Ferguson VMG, Spalton DJ. Recovery of the blood-aqueous barrier after cataract surgery. Br J Ophthalmol 1991;75:106-10. 